\title{
ESTRUCTURAS ECONÓMICAS- SOCIALES Y POBREZA EN LA ENAHO 2004-2007 DE LA REGIÓN JUNÍN ${ }^{\dagger \dagger \dagger \dagger \dagger ~}$
}

Saúl Darío Díaz Maraviłłłł‡, Melitón Vílchez Perales

Facultad de Economía de la Universidad Nacional del Centro del Perú

\begin{abstract}
RESUMEN
En el presente trabajo se ha tratado de consolidar algunos datos del ENAHO 2004-2008 referidos a condiciones de la estructura económica y social, así como la situación de la pobreza de la región Junín. En la primera parte que corresponde al marco teórico se trata de algunas definiciones que realizan para elaborar los índices de pobreza según los resultados de la Enaho. En la segunda parte se trata los resultados y la discusión de los mismos donde se presentan las estructuras productivas, sociales y de pobreza de la región Junín. En ellas se encuentra que la vocación productiva de la región es una vocación agrícola y que la industria tiende a disminuir, pero también el sector servicios se encuentra en una fase creciente cada vez más en la región Junín. Asimismo, la región Junín presenta un grado de desarrollo medio bajo $($ IDH $=0,592)$ y casi la mitad de la población vive en la pobreza, así como también de cada 100 personas, 13 están en situación de pobreza extrema. Estos nos dan como conclusión que es coincidente la situación de la estructura económica con la situación de pobreza. Si la mayoría se dedica a la agricultura, que es un sector de pobreza asentada en la zona rural. Por eso, los distritos más rurales tienen altas tasa de desnutrición y de pobreza extrema. De allí que la caracterización de la estructuras económicas en Junín se encuentran en estado inicial (agricultura de sobrevivencia, pero sin embargo ya también tenemos que el sector terciario está en una etapa de tendencia creciente y por otro lado la industria está en tendencia decreciente. Es eso que los teóricos llama desarrollo espurio o la terciarización de la economía, sin haber desarrollado plenamente la segunda fase o sector industrial.
\end{abstract}

Palabras clave: Estructuras socioeconómicas, pobreza, desnutrición

\section{ECONOMIC AND SOCIAL STRUCTURES AND POVERTY IN THE ENAHO 2004-2007 ENAHO JUNIN REGION}

\begin{abstract}
In this paper we have tried to consolidate some data from ENAHO 2004-2008 relating to conditions of economic and social structure, and the situation of poverty in the Junin region. In the first part that corresponds to the theoretical framework of gaps done to develop definitions about the poverty rates based on the results of the Enaho. In the second part discusses the results and discussion of these structures which are productive, social and poverty in the Junin region. They found that the production profile of the region is an agricultural use and that the industry tends to decrease, but also the services sector is in an increasingly growing phase in the Jenin region. Also, the Junin region has a low average level of development $(\mathrm{HDI}=$ 0.592) and almost half the population is poor, as well as that from each100 people, 13 are in extreme poverty. These give us the conclusion that the situation is coincident economic structure with poverty. If the majority is devoted to agriculture, a sector that is seated poverty in rural areas. Therefore, more rural districts have high rates of malnutrition and extreme poverty. Hence, the characterization of the economic structures in Junín are in initial state (agriculture for survival, and yet we also have the tertiary sector is in a stage of growing trend on the other hand the industry is declining. It development theorists called spurious or service economy, without having fully developed the second phase or industrial sector.
\end{abstract}

Key Words: socio-economic structures, poverty, malnutrition.

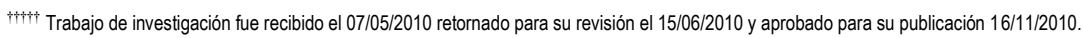




\section{INTRODUCCIÓN}

La Encuesta Nacional de Hogares (ENAHO) se viene realizando desde el año 2004. Esta encuesta que abarca a más de 22 mil hogares de todo el Perú con un muestreo aleatorio polietápico en zig-zag a lo largo de todo el territorio nacional extrae información de los hogares respecto a todos los órdenes de la vida de una familia. Desde el tipo de vivienda en que habitan hasta la percepción de gobernabilidad en el momento de la encuesta. Esta información que se puede consolidar a nivel de regiones no está siendo utilizada por los centros investigación, especialmente por las universidades, pese a que insume enormes costos por parte del Estado Peruano. Por lo que, nosotros estamos tratando de consolidar la información desde una perspectiva socioeconómica y de pobreza. Por el lado social se tendrá indicadores de vivienda, educación y salud. Por el lado económico estará básicamente el nivel de gastos de las familias y la producción o tipos de actividades que desarrollan. Por el lado de la pobreza se enmarcará básicamente en las necesidades básicas insatisfechas (NBI) y la línea de pobreza (LP), que ira estrictamente a corroborar la reciente presentación del MAPA DE POBREZA 2007 que hizo el INEI. Por eso los objetivos de la investigación fueron: "Establecer las determinantes de las estructuras económicas y sociales y la generación de la pobreza en la Región Junín". Además de todo lo anterior la investigación sirve para aplicar ciertos paquetes estadísticos en la obtención de datos, que por lo demás los datos en el Perú están sueltos o listos para ser usados, pero muy pocos están sacando provecho de los mismos. Esto teniendo en cuenta que la pobreza es un mal endémico y que suscita muchas controversias, tanto por el lado de las instituciones gubernamentales, como en el campo político, pero que realmente no se está haciendo lo necesario para erradicarlo. Precisamente si observamos el portal de la SNMPE, un ente empresarial ligado a los grupos de poder, vemos que con bombos y platillos sostienen que la minería ha reducido la pobreza en los llamados "distritos mineros", pero el ultimo mapa de la pobreza del INEI lo contradice todo y basta visitar in situ los centros mineros para comprobar que pese al boom de la minería en estos diez últimos años la pobreza en muchos caso casi nada se ha resuelto. De allí que este trabajo merece la importancia del caso para entender de cómo a través de la obtención de datos por parte de un organismo estatal se puede hacer investigación.

\section{MATERIALES Y MÉTODOS}

\section{Material}

Entre los materiales utilizados se encuentran los bibliográficos, los archivos estadísticos, un ordenador electrónico para el procesamiento de la información y la encuesta de la ENAHO 2004-2008.

\section{Método de investigación}

En la elaboración de la presente investigación se está empleando el método científico que presenta las siguientes características:

- Implica siempre la abstracción de un fenómeno o porción de la realidad.

- La elaboración de afirmaciones de tipo general.

- El apoyarse en afirmaciones o datos de carácter empírico en relación a los planteamientos hechos.

- El ceñirse a los hechos y no a los valores y al deber ser. Es decir, los hechos como son y no como deberían.

- La objetividad en la descripción de los fenómenos.

\section{RESULTADOS}

\section{La estructura económica}

La actividad agrícola tiene una tendencia creciente y la actividad industrial (manufactura) una tendencia decreciente. Igualmente la tendencia es creciente para el rubro otros servicios (como educación, finanzas, etc.).

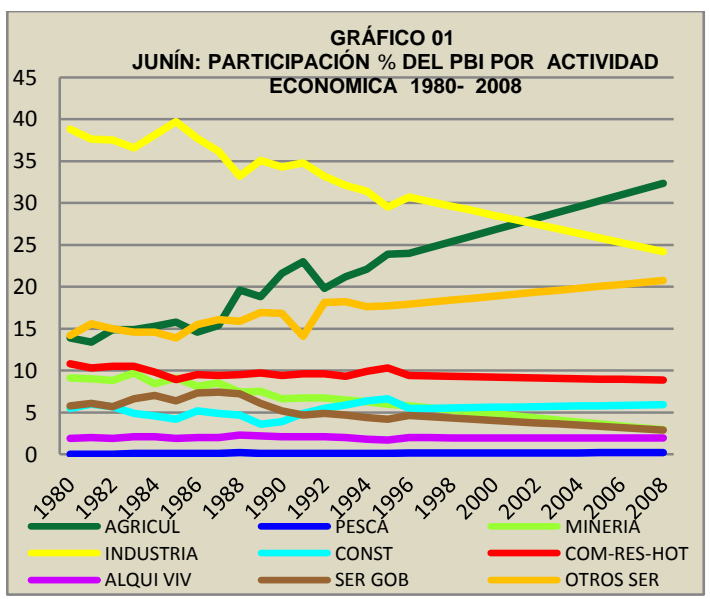

Entre el año 2000 y 2002 se da el quiebre entre la agricultura y la industria. Si en Junín antes del 2000 predominaba la actividad manufactura (como dijimos anteriormente influida por dos grandes empresas (como la Doe Run y Cemento Andino) a partir del 2002 la actividad predominante es la agricultura.

\section{La Estructura Educativa}

\section{POBLACIÓN ESCOLAR}

Según los resultados de la Encuesta Nacional de Hogares 2006 (ENAHO), el 83,4\% de la población escolar de 3 a 16 años de edad en el departamento de Junín que asiste a un centro o programa regular de enseñanza es atendido por el sector estatal, mientras que sólo el $10,9 \%$ asistió a un 
centro educativo no estatal. La importancia de la educación radica en tanto constituye el pilar para la formación de capital humano, proporciona los medios necesarios para la realización personal y contribuye con la sociedad aportando personas capacitadas para el desarrollo del país. Lo que aquí no se indica es el desnivel que hay entre educación estatal y educación no estatal o privada.

\section{TASA DE MATRÍCULA Y ASISTENCIA ESCOLAR}

Según la información captada por la Encuesta Nacional De Hogares 2006 (ENAHO), la matrícula escolar alcanzó una tasa promedio de $85,9 \%$ en el departamento de Junín. La tasa de matrícula escolar de mujeres fue ligeramente mayor $(86,2 \%)$ en comparación con la tasa de varones, que fue de $85,6 \%$.

\section{ATRASO ESCOLAR}

En el departamento de Junín la tasa de atraso escolar educativa de primaria, es ligeramente mayor para los niños de 6 a 11 años de edad (28,5\%), frente al porcentaje de las niñas del mismo grupo etáreo $(26,2 \%)$. En el nivel de educación secundaria, es decir, la población de 12 a 16 años, tuvo un atraso escolar mayor en los hombres $(28,2 \%)$ que en las mujeres $(25,2 \%)$.

\section{AÑOS PROMEDIO DE ESTUDIO}

Los años promedio de estudio alcanzado por la población, es interpretado como un indicador que mide el acceso efectivo de la población al sistema educativo e indica su efectividad a largo plazo.

Este indicador refleja sólo la cantidad de educación recibida y no la calidad de la misma. Según los resultados de la Encuesta Nacional Continua, en el año 2006 en el departamento de Junín, la población de 15 y más años de edad, logró 9,6 años en promedio de estudios, este valor fue mayor para el área urbana (10,5 años), mientras que para el área rural (7,5 años) fue menor en 3,0 años. El promedio de años de estudios alcanzado, es ligeramente mayor en los varones (9,9 años), que en las mujeres (9,3 años), notándose que cada vez la diferencia entre los sexos, es menor.

\section{NIVEL DE EDUCACIÓN ALCANZADO}

El nivel de educación alcanzado por la población del departamento de Junín, es un indicador de logro educativo. En el año 2006, el 42,7\% de la población de 15 y más años de edad alcanzó a estudiar algún año de educación secundaria, el $26,1 \%$ algún año de educación primaria, el $23,8 \%$ tiene educación superior $(11,7 \%$ superior no universitaria y $12,1 \%$ superior universitaria), y el $7,4 \%$ sólo tiene nivel inicial o carece de nivel educativo alguno.

\section{La estructura de salud}

Padecimiento de alguna enfermedad o malestar crónico Los resultados de la Encuesta Nacional Continua (ENAHO), correspondiente al 2006, indican que el padecimiento de alguna enfermedad o malestar crónico (Comprende: artritis, hipertensión, asma, reumatismo, diabetes, tuberculosis, VIH, colesterol, depresión, etc.) En el departamento de Junín, es la población que reporta haber padecido alguna enfermedad en mayor proporción son las mujeres $(11,9 \%)$ y varones con $(9,1 \%)$. Según grupos de edad con mayor proporción de padecimiento de enfermedades son de 65 a más años $40,0 \%$.

\section{Consumo de bebidas alcohólicas}

En el departamento de Junín el consumo de bebidas alcohólicas al menos una vez en la semana anterior a la encuesta, la ENAHO 2006, muestra el comportamiento siguiente: Un poco más de la cuarta parte de la población del departamento de Junín de 12 y más años de edad, ha consumido alcohol (25,5\%). Los hombres mencionan haberlo hecho en una proporción de más del doble $(35,8 \%)$, respecto a las mujeres $(15,4 \%)$.

\section{La desnutrición}

La pobreza siempre comienza y termina con la desnutrición y es el signo visible de la misma. Es decir las personas son desnutridas porque son pobres y la desnutrición a la larga va a generar pobres (aunque no siempre) en la mayoría de los casos, ya que una persona desnutrida tiene mayores probabilidades de no poder ejercer sus capacidades, por lo que siempre termina en la pobreza.

La mayor tasa de desnutrición crónica se da en San Juan de Iscos $(54,55 \%)$ seguido de Chongos Alto (53,55\%). Es decir de cada 10 personas, entre 5 ó 6 están desnutridas. En este primer quintil hay 10 distritos que pertenecen a la provincia de Huancayo.

Entre todos los distritos se puede establecer el rango entre el último y el primero y este es igual a $36,28 \%$ que está muy cerca del promedio nacional que es igual a $38,73 \%$.

En el último quintil o quinto quintil, están los distritos donde de cada 10 personas 2 ó 3 están con desnutrición crónica. El distrito menos desnutrido es San Ramón, seguido de Chanchamayo, ambos pertenecientes a la Selva Alta.

Entre todos los distritos se puede establecer el rango entre el último y el primero y este es igual a $36,28 \%$ que está muy cerca del promedio que es igual a $38,73 \%$.

En el siguiente cuadro, se presenta la desnutrición por 
provincias según el patrón NCHS y el patrón OMS.

Tabla 01.- Junín: desnutrición crónica según patrón por provincias

\begin{tabular}{lcccc}
\hline & \multicolumn{2}{c}{ PATRÓN NCHS } & \multicolumn{2}{c}{ PATRÓN OMS } \\
\cline { 2 - 5 } & $(\%)$ & ABS. & $(\%)$ & ABS. \\
\hline JUNÍN DEPARTAMENTO & 26,20 & 32780 & 31,90 & 39911 \\
HUANCAYO & 31,03 & 13451 & 33,12 & 14358 \\
CONCEPCIÓN & 35,93 & 2260 & 42,99 & 2704 \\
CHANCHAMAYO & 18,41 & 3386 & 26,12 & 4804 \\
JAUJA & 27,49 & 2388 & 37,55 & 3262 \\
JUNÍN & 20,70 & 529 & 35,45 & 907 \\
SATIPO & 40,60 & 10532 & 37,68 & 9777 \\
TARMA & 17,61 & 1760 & 32,87 & 3285 \\
YAULI & 7,78 & 377 & 27,03 & 1312 \\
CHUPACA & 29,07 & $\mathbf{1 4 6 9}$ & $\mathbf{4 3 , 5 9}$ & $\mathbf{2 2 0 4}$ \\
\hline \multicolumn{4}{c}{ Fuente: ENAHO } & $\mathbf{2 0 0 4 - 2 0 0 8}$
\end{tabular}

Según esto se tiene que la provincia de Chupaca es la que tiene mayor tasa de desnutrición crónica.

\section{LA ESTRUCTURA DE POBREZA}

La pobreza tiene una estructura entre el total de pobres y la pobreza extrema. El total de pobres son aquellos que tienen ingresos por debajo de una línea de pobreza constituido generalmente por la canasta básica de consumo. Es decir, el valor de la canasta básica de consumo es la línea que separa entre los pobres y no pobres. En cambio la pobreza extrema es aquella que está por debajo de una canasta alimentaria o cierta cantidad de nutrientes establecidos por la OMS. Generalmente la canasta alimentaria es un tercio del valor de la canasta básica de consumo.

La provincia con más pobreza extrema es Concepción (con $24 \%$ ), en tanto la que tiene menos pobreza extrema es Yauli, considerado un emporio minero metalúrgico (4\%). En promedio entre las provincias hay un $15,53 \%$ de pobreza extrema muy por encima del promedio departamental que es $13,4 \%$. Asimismo, hay seis provincias que están por encima del promedio departamental (Concepción, Satipo, Junín, Chupaca, Jauja y Tarma), mientras que sólo tres provincias están por debajo del índice departamental estás son: Chanchamayo, Huancayo y Yauli.

\section{DISCUSIÓN}

\section{Sobre la estructura productiva}

La región Junín es esencialmente agrícola. La actividad económica gira en torno a la agricultura. Mayormente se concentra en la Selva relacionada a los frutales (piña, cítricos café, etc.). La tendencia ha sido creciente y el otro sector que ha disminuido su participación ha sido el sector industria. Es decir, cada año que pasaba la región Junín se volvía más agrícola y menos industrial. Por otro lado se destierra el mito de que Junín es Minero. Podría ser "metalúrgico", pero la actividad minera es muy inferior al sector agrario y al sector industrial. La industria de la metalurgia y de minería está fuertemente influenciada por la presencia de la Doe Run en La Oroya y por Cemento Andino en el distrito de La Unión (Tarma). En el caso de la Doe Run, su influencia y su impacto en la zona ha logrado ser positivo ya que en los distritos donde se asienta y sus alrededores (distritos de más directa influencia) se encuentran entre los menos pobres y los más desarrollados (la Oroya tiene el IDH más alto de la región: 0,635) y apenas tiene un $1,5 \%$ de pobreza extrema, además de tener el ingreso promedio mensual per cápita más alto de la región.

En cuanto a desarrollo se refiere, la región Junín presenta un desarrollo medio bajo (IDH= 0,592) y se encuentra en el puesto del ranking de departamentos a nivel nacional. Esto coincide con la estructura productiva porque si es desarrollo bajo, se debe a que mayormente la PEA se encuentra en el sector agrícola y no se tiene un buen capital humano. Asimismo es coincidente con el nivel de pobreza extrema que la Región Junín tiene en un 13,4\%.

\section{Sobre la estructura social}

En la esfera social, ésta estructurada en base al sector público. Es decir, la vida de la sociedad en mayor parte está en función al aparato público. Por ejemplo, el $80 \%$ de las personas en edad escolar asisten a una entidad educativa pública. El $70 \%$ de las personas se atienden en aspectos relacionados a la salud en instituciones públicas. El mayor empleador sigue siendo la administración pública, así como los mejores empleos (empleos de calidad: cuentan con planillas, seguridad social y gozan de vacaciones). El sector gubernamental presenta un tercio de la actividad económica de la región, de allí que también se nota un avance creciente del sector público. Sobre el impacto de la actividad minera tan promovida en estos tiempos se nota muy poco dentro de la región Junín. En general la presencia del sector privado de gran magnitud, salvo la Doe Run en la Oroya, es muy débil en la región Junín. Por ejemplo el caso de Cemento Andino es bien claro al respecto. La que el distrito donde está ubicado Cemento Andino es la Unión (Tarma), y tiene los siguientes indicadores: IDH=0,584; Desnutrición 33,1\%; Pobreza extrema: 10,8\%; Pobreza relativa: $50,0 \%$. Esto quiere decir a pesar de que la empresa extrae el recurso mineral para fabricar cemento y que pertenecen a las comunidades campesinas de La Unión, este distrito tiene a su población en pobreza y bastante mal nutrida. Un claro ejemplo de que el sector privado sobre todo en la minería e industria minera no contribuye al desarrollo de los pueblos y sólo sirve para sacar sus recursos, depredarlos y la pobreza se mantiene perenne. 


\section{Sobre la estructura de pobreza}

En la región Junín, se tienen los siguientes indicadores: $\mathrm{IDH}=0,592$; ranking $=10$ a nivel nacional y entre departamentos; Pobreza relativa $=43,0 \%$; Pobreza extrema $=13,4 \%$; Ingreso mensual promedio per cápita $=$ 318,2 nuevos soles. Esto quiere decir que se encuentra en el desarrollo medio bajo, casi la mitad de la población se encuentra en pobreza y de cada cien personas 13 personas se encuentran en pobreza extrema. Además existen varios distritos muy pobres que bordean más de un tercio de pobreza extrema a pesar de que en la región existen enormes recursos en sus regiones naturales (región Sierra y región Selva). Esta cerca de Lima y tiene bastante infraestructura. Por lo demás la pobreza resultante debe tener otras causas que no explicamos en esta investigación.

\section{CONCLUSIONES}

- La economía regional está influenciada en mayor medida por el sector público y por una creciente tercerización económica.

- Pese al crecimiento sostenido la pobreza no se ha erradicado de manera sustancial. Junín sigue siendo pobre a pesar de los enormes recursos que posee.

- Hay enormes brechas sociales que todavía no se resuelven pese a la cada vez más presencia estatal.

\section{REFERENCIAS BIBLIOGRÁFICAS}

- Moore, Barrington. Orígenes de las dictaduras y democracias, Edit. Aguilar, España. 1974.

- Corporación Departamental de desarrollo de Junín. Diagnóstico Departamental de Junín, Huancayo, 1988.

- CENTROMIN PERU. Área de influencia de CENTROMIN PERU, La Oroya, 1978

- Indacochea, Alejandro. Diamante de Competividad de la Región Junín, Huancayo, 2002. 